\title{
Sequential testing effects and the relationship between recognition and recognition failure
}

\author{
MICHAEL S. HUMPHREYS and PAUL A. BOWYER \\ Northwestern University, Evanston, Illinois 60201
}

\begin{abstract}
Tulving and Wiseman (1975) reported that there was a systematic relationship between the proportion of words recognized and the proportion of recallable words recognized. This relationship indicates a moderate positive covariation between recognition and recall across subject items, when each subject is given both types of test, recognition followed by recall. In this paper it is shown that the theoretical enterprise of trying to account for this relationship is fruitless unless the data are corrected for sequential testing effects. Evidence on the existence of these effects is reviewed, and then it is shown how they introduce a measure of dependency between recognition and recall. When the data are corrected, the theories proposed by Begg (1979) and Flexser and Tulving (1978) are shown to be poorly supported. The utility of the general enterprise of determining the relationship between recognition and recall by these means is also questioned.
\end{abstract}

Tulving and Wiseman (1975) first reported that there was a systematic relationship in studies employing the "recognition failure" paradigm between the proportion of words recognized $P(R n)$ and the proportion of recallable words recognized $\mathrm{P}(\mathrm{Rn} / \mathrm{Rc})$. Reviewing the results from several experiments, Tulving and Wiseman concluded that this relationship could be adequately described by the equation $P(R n / R c)=P(R n)+c[P(R n)-$ $\left.P(R n)^{2}\right]$, where $c$ was estimated to be .5 . These predicted values of $P(R n / R c)$ are all somewhat greater than would be expected if recognition and recall were stochastically independent [i.e., $\mathrm{P}(\mathrm{Rn})=\mathrm{P}(\mathrm{Rn} / \mathrm{Rc})$ ], indicating a moderate amount of dependency between recognition and recall. In all of these studies, the subjects had first studied a list of word pairs and then were tested successively, first for recognition and then for recall. On the recall test the subjects were provided one member of a pair as a cue and were asked to recall the words they had just recognized. Flexser and Tulving (1978) looked at the results of several additional studies and concluded that the relationship first noted by Tulving and Wiseman was largely invariant over a variety of experimental procedures, conditions, and materials.

This invariance in the relationship between $P(R n)$ and $\mathrm{P}(\mathrm{Rn} / \mathrm{Rc})$ has provided a "puzzle" that several theorists have attempted to explain. Begg (1979) has reviewed these explanations and has classified them into two classes. One class, represented by Begg (1979) and Flexser and Tulving (1978), starts from the assumption that at the retrieval level recognition and recall are

Portions of this work were presented at the 1978 meetings of the Psychonomic Society in San Antonio, Texas. Requests for reprints should be sent to Michael S. Humphreys, Department of Psychology, University of Queensland, St. Lucia, Queensland 4067, Australia. independent. They then introduce some dependency in order to fit the Tulving and Wiseman (1975) empirical function.

The alternative class of theories starts with the assumption that recognition and recall are basically the same process and thus dependent (see Jones, 1978; Kintsch, 1978; Martin, 1975; Reder, Anderson, \& Bjork, 1974). These theories then make specific assumptions in order to reduce the degree of dependency between recognition and recall. The effort by these theorists has been to show that their theories can account for recognition failure, although they have not specifically tried to fit the Tulving and Wiseman (1975) function. In this paper we are not directly concerned with the question of which theory or class of theories is most likely correct. Instead, our contention is that the Tulving and Wiseman function is not the appropriate function to fit. Specifically, we will show that sequential testing effects result in a subset of items that are both recalled and recognized. Thus, a degree of dependency is introduced by the very nature of the paradigm (the recognition test necessarily precedes the recall test). Unless this degree of dependency is corrected for, the theoretical enterprise of trying to determine the relationship between recognition and recall is fruitless.

In our examination of the effect sequential testing has on the recognition-recall relationship, it also becomes apparent that determining this relationship is going to be an exceedingly difficult, if not impossible, task. Thus, the utility of the general enterprise is also questioned.

\section{SEQUENTIAL TESTING EFFECTS}

In this paradigm subjects study a pair of words, $A B$, are tested for recognition of some of the words, and 
are then tested for recall of the same words. Some experiments have tested both the A and B words for recognition and recall. However, in the remainder of this paper, we present the experiments as if only the B words are tested for recognition and the $A$ words are provided as cues for the recall of the B words. Recall is said to be "primed" if the B word corresponding to a particular A word was present on the recognition test and "unprimed" if it was not. A "priming effect" is defined as the difference in the proportion recalled between primed $(R c)$ and unprimed (URc) recall $[P(R c)-P(U R c)=\Delta]$.

In Table 1 the difference between primed and unprimed recall is shown for 16 conditions in 10 different experiments (see column headed " $\Delta$ "). Out of the
16 conditions, there were 2 instances in which there was a nonsignificant negative priming effect, 2 instances with no priming effect, 3 instances with a nonsignificant positive priming effect, and 9 instances with a significant positive priming effect $(\mathrm{p}<.05)$. In addition, there were seven conditions in Begg (1979, Experiment 3), all of which showed a positive priming effect. (The results from this experiment are shown in Table 2 and will be discussed separately.) Thus, there seems to be little question that positive priming effects are the rule and not the exception. Note that, as Bowyer and Humphreys (1979) have pointed out, there is a strong positive correlation (across experiments) between $\Delta$ and $\mathrm{P}(\mathrm{Rn})$. Over the 16 conditions in Table 1, this correlation is .86 . Furthermore, the average proportion recog-

Table 1

The Proportion Recognized [P(Rn)], Unprimed Recall [P(URc)], the Priming Effect ( $\Delta)$, the Observed Proportion of Recognition Conditional on Recall [P(Rn/Rc)], and the Predicted Proportion of Recognition Conditional on Recall from the Priming Effect and From the Tulving and Wiseman Empirical Equation for 16 Conditions in 10 Experiments

\begin{tabular}{|c|c|c|c|c|c|c|c|}
\hline \multirow[b]{2}{*}{ Experiment } & \multirow[b]{2}{*}{ Condition } & \multirow[b]{2}{*}{$P(R n)$} & \multirow[b]{2}{*}{ P(URc) } & \multirow[b]{2}{*}{$\Delta$} & \multirow[b]{2}{*}{$\mathrm{P}(\mathrm{Rn} / \mathrm{Rc})$} & \multicolumn{2}{|c|}{ Prediction } \\
\hline & & & & & & Priming** & EPt \\
\hline Postman (1975) & $\begin{array}{l}\text { HA } \\
\text { MA }\end{array}$ & $\begin{array}{l}.50 \\
.46\end{array}$ & $\begin{array}{l}.35 \\
.19\end{array}$ & $\begin{array}{l}.03 \\
.05\end{array}$ & $\begin{array}{l}.53 \\
.58\end{array}$ & $\begin{array}{l}.54 \\
.58\end{array}$ & $\begin{array}{l}.62 \\
.59\end{array}$ \\
\hline $\begin{array}{l}\text { Wiseman \& Tulving (1976) } \\
\text { Experiment } 1 \\
\text { Experiment } 2 \\
\text { Experiment } 4\end{array}$ & $\begin{array}{l}\text { Related } 1: 7 \mathrm{RC} 24 \\
\text { Unrelated } 1: 7 \mathrm{RC} 24\end{array}$ & $\begin{array}{l}.26 \\
.18 \\
.59 \\
.45\end{array}$ & $\begin{array}{l}.59 \\
.34 \\
.60 \\
.35\end{array}$ & $\begin{array}{r}.00 \\
-.08 \\
.09 \\
-.01\end{array}$ & $\begin{array}{l}.33 \\
.24 \\
.68 \\
.58\end{array}$ & $\begin{array}{l}.26 \\
.64\end{array}$ & $\begin{array}{l}.36 \\
.25 \\
.71 \\
.57\end{array}$ \\
\hline Humphreys (1978) & & .78 & .43 & $.16^{*}$ & .87 & .84 & .86 \\
\hline $\begin{array}{l}\text { Bowyer \& Humphreys (1979) } \\
\text { Experiment } 1 \\
\text { Experiment } 2\end{array}$ & $\begin{array}{l}\text { A Words } \\
\text { B Words } \\
\text { A Words } \\
\text { B Words }\end{array}$ & $\begin{array}{l}.84 \\
.71 \\
.80 \\
.63\end{array}$ & $\begin{array}{l}.48 \\
.68 \\
.35 \\
.56\end{array}$ & $\begin{array}{l}.18^{*} \\
.00 \\
.17^{*} \\
.08^{*}\end{array}$ & $\begin{array}{l}.93 \\
.77 \\
.91 \\
.73\end{array}$ & $\begin{array}{l}.88 \\
.71 \\
.86 \\
.67\end{array}$ & $\begin{array}{l}.91 \\
.81 \\
.88 \\
.74\end{array}$ \\
\hline Humphreys \& Bowyer (Note 1) & $\begin{array}{l}\text { Old Only } \\
\text { Old New }\end{array}$ & $\begin{array}{l}.86 \\
.84\end{array}$ & $\begin{array}{l}.65 \\
.61\end{array}$ & $\begin{array}{l}.12^{*} \\
.12^{*}\end{array}$ & $\begin{array}{l}.94 \\
.92\end{array}$ & $\begin{array}{l}.89 \\
.86\end{array}$ & $\begin{array}{l}.92 \\
.91\end{array}$ \\
\hline Bowyer (1977) & & .81 & .32 & $.19 *$ & .91 & .88 & .89 \\
\hline Beg $(1979$, Experiment 1$)$ & $\begin{array}{l}\text { Meaningful } \\
\text { Rote }\end{array}$ & $\begin{array}{l}.76 \\
.61\end{array}$ & $\begin{array}{l}.48 \\
.15 \\
\end{array}$ & $\begin{array}{l}.10^{*} \\
.08^{*}\end{array}$ & $\begin{array}{l}.79 \\
.84 \\
\end{array}$ & $\begin{array}{l}.80 \\
.75 \\
\end{array}$ & $\begin{array}{l}.85 \\
.73 \\
\end{array}$ \\
\hline
\end{tabular}

Note-The abbreviations used for conditions are taken from the original articles. $\quad * p<.05 . \quad * *$ The predicted value of $P(R n / R c)$ based on the magnitude of the priming effect. TThe Tulving and Wiseman (1975) empirical prediction $P(R n / R c)=P(R n)+$ $.5\left[P(R n)-P(R n)^{2}\right]$.

Table 2

The Proportion Recognized [P(Rn)], Unprimed Recall [P(URc)], Primed Recall [P(Rc)], Proportion of Words Recalled Conditional on Nonrecognition $[P(R c / \bar{R} n)]$, the Proportion Recognized Conditional on Recall $[P(R n / R c)]$, and the Estimate of the Proportion Recognized Conditional on Unprimed Recall [P(Rn/URc)], from Begg (1979)

\begin{tabular}{|c|c|c|c|c|c|c|c|}
\hline \multicolumn{2}{|c|}{ Condition } & \multirow{2}{*}{$\begin{array}{c}P(\mathrm{Rn}) \\
.56 \\
.43\end{array}$} & \multirow{2}{*}{$\begin{array}{c}\text { P(URc) } \\
.004 \\
.05\end{array}$} & \multirow{2}{*}{$\begin{array}{c}\mathrm{P}(\mathrm{PRc}) \\
.02 \\
.08\end{array}$} & \multirow{2}{*}{$\begin{array}{c}\mathrm{P}(\mathrm{PRc} / \overline{\mathrm{Rn}}) \\
.03 \\
.06\end{array}$} & \multirow{2}{*}{$\begin{array}{c}\mathrm{P}(\mathrm{Rn} / \mathrm{Rc}) \\
.28 \\
.59\end{array}$} & \multirow{2}{*}{ 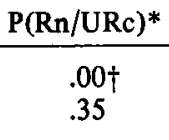 } \\
\hline List 1 & $\begin{array}{l}\text { Separate } \\
\text { Joine }\end{array}$ & & & & & & \\
\hline List 2 & $\begin{array}{l}\text { Separate } \\
\text { Joint }\end{array}$ & $\begin{array}{l}.69 \\
.49\end{array}$ & $\begin{array}{l}.02 \\
.09\end{array}$ & $\begin{array}{l}.04 \\
.17\end{array}$ & $\begin{array}{l}.02 \\
.14\end{array}$ & $\begin{array}{l}.86 \\
.58\end{array}$ & $\begin{array}{l}.72 \\
.20\end{array}$ \\
\hline Repeated & $\begin{array}{l}\text { Separate } \\
\text { Mixed } \\
\text { Joint }\end{array}$ & $\begin{array}{l}.88 \\
.78 \\
.64\end{array}$ & $\begin{array}{l}.02 \\
.19 \\
.40\end{array}$ & $\begin{array}{l}.04 \\
.27 \\
.42 \\
\end{array}$ & $\begin{array}{l}.04 \\
.24 \\
.42 \\
\end{array}$ & $\begin{array}{l}.88 \\
.81 \\
.64 \\
\end{array}$ & $\begin{array}{l}.76 \\
.73 \\
.62 \\
\end{array}$ \\
\hline
\end{tabular}

*Estimated from Equation 2. TThe predicted value is actually less than .00 . 
nized in the conditions in which nonsignificant priming effects were found was .45 , compared with .77 in the conditions in which significant priming effects were found. Thus, most of the failures to find significant positive priming effects are probably due to the small levels expected with the low levels of recognition in these conditions (see Bowyer \& Humphreys, 1979, for an extended discussion of this issue).

\section{PRIMING AND THE RELATIONSHIP BETWEEN $\mathbf{P}(\mathbf{R n})$ AND P(Rn/Rc)}

\section{Can Unrecognized Words Be Primed?}

With one exception, those authors who have discussed priming effects have concluded that presentation of a word on the recognition test will not affect its subsequent cued recall if the word is not recognized (Begg, 1979; Bowyer \& Humphreys, 1979; Wiseman \& Tulving, 1976). This assumption has apparently struck most researchers and theorists in this area as being intuitively reasonable, although relatively little evidence on this issue exists. Some of the evidence that does exist has been provided by Bowyer and Humphreys (1979). They pointed out that, across experiments, there was a substantial positive correlation between $\mathrm{P}(\mathrm{Rn})$ and $\Delta$. They also looked at item correlations within experiments. Using a multiple-regression analysis, they found that the probability of recognizing a word was significantly correlated with the probability of primed recall but not with that of unprimed recall. Furthermore, the probability of recognition was a better predictor of priming than was the probability that the to-be-recognized word could be used as a cue to recall the other member of the study pair. These correlational results suggest that there is a strong relationship between recognition and priming, but they cannot establish that recognition is necessary for priming.

Vining and Nelson (1979) presented data that they interpreted as showing that learning of both recognized and unrecognized words can occur on a test trial. In their experiments they omitted the study trial on the $A B$ pairs and gave a recognition test on the $B$ terms that was followed by a cued recall test. The targets were weak associates of the recall cues, although they had never been paired with the cues. As a cover story, the subjects were told that they were in an ESP experiment in which a telepath was trying to send them the correct answers. On the recall trial the subjects were told that the words they were supposed to recall had been on the recognition test and were encouraged to guess from those items. They of course had no idea which of the words on the recognition test they were supposed to recall, although in Experiment 2 they might have assumed that the to-be-recalled targets were related to the cues in some fashion, and they were explicitly informed about this cue-target relationship in Experiment 3. On the cued recall test, the subjects recalled $10 \%$ and $13 \%$ of the targets in two different experiments. More important for the purpose of this discussion, they recalled $9 \%$ and $4 \%$ of the targets they had not recognized. Our interpretation is that, when encouraged to do so, subjects can recall words from the recognition test. In the Vining and Nelson experiment, they were not required to match the recalled targets with the cues, and there was no reason for them to limit the words they recalled to those they recognized.

Under these conditions it is not surprising that the subjects recalled both recognized and unrecognized words. Under the standard conditions obtaining in these experiments, on the other hand, subjects are asked to recall only words that are actually presented on the study list and to match these words with the appropriate cue. With these instructions, there is very little effect of a word's simply being present on the recognition test. Humphreys (1978) selected two weak associates for each cue $\left(A-B_{1}, B_{2}\right)$. Subjects studied one of the cuetarget pairs (e.g., $A \cdot B_{1}$ ). For some pairs, $B_{2}$, not $B_{1}$, was tested for recognition. For these pairs the rate at which $B_{2}$ intruded on the subsequent cued recall test could be determined. Ignoring correct recalls $\left(B_{1}\right.$ recalled), there were 216 opportunities for an intrusion to occur, and there were exactly six intrusions of the $B_{2}$ responses. At least one of these six intrusions was probably due to the similarity between $B_{1}$ and $B_{2}$, as this word had been falsely recognized on the recognition test. In a somewhat similar experiment, Humphreys and Bowyer (Note 1) provided recall cues for the distractors on the recognition test as well as for old targets. In this experiment, there were 576 opportunities to recall a target that had only been present as a distractor on the recognition test. There were exactly two instances in which such a target was recalled.

In considering how priming would affect the relationship between $P(R n)$ and $P(R n / R c)$, we will go beyond the data in making the strong assumption that if $B$ is present on a recognition test and it is unrecognized, there will be no net change in the probability of recalling $B$ given $A$ as a cue. There of course may be generalized interference (see Postman, 1975), but the effect on AB should be no greater than the effect on any other pair. Although this may not be true, it should be noted that this assumption is critical to much of the theoretical thinking of Tulving and his associates (Tulving, 1976; Tulving \& Thomson, 1973; Watkins \& Tulving, 1975; Wiseman \& Tulving, 1976). For example, Wiseman and Tulving (1976, p. 357) state, "Should this assumption be shown to be untenable, we would have to revise our thinking about the theoretical significance of recognition failure of recallable words in these and other experiments."

\section{Theoretical Consequences}

In analyzing the effect of the recognition test on recall, we will consider separately the effects of the study trial on the $\mathrm{AB}$ pair and the effects of the recog. 
nition test on B. As a result of the study trial, some of the pairs will be learned and some will not. They are learned in the sense that they would have been recalled even if they had not been present on the recognition test. Perhaps the best way to think of this is that, following the study trial, there is a distribution of item strength. Some of the strengths are sufficient so that, whatever the generalized interference effects of the recognition test, these items will still be recalled. We can express this idea by the assertion that with probability $a$, an item will enter the learned state $\left(\mathrm{L}_{S}\right)$ after the pair has been studied. With probability $1-a$, the item will enter the unlearned state $\left(U_{S}\right)$. By the definition of State $\mathrm{L}_{\mathrm{S}}$, we can estimate $a$ by the probability that an unprimed item is recalled [P(URc)] .

When we test an item for recognition before testing for recall, we can no longer determine whether any given item was in State $\mathrm{L}_{S}$ or State $\mathrm{U}_{S}$. The problem here is that an item could be recalled either because it was leamed on the study trial (entered State $L_{S}$ ) or because it was present on the recognition test. We can, however, write down in a completely general manner expressions for the probability that an item will be recallable following the recognition test as a function of whether the item was in State $L_{S}$ or State $U_{S}$ following the study trial and whether the item was recognized $\left(R_{n}\right)$ or not recognized $\left(\bar{R}_{n}\right)$. An item that is recallable following the recognition test is said to be in State $L_{R}$, and one which is not recallable, in State $U_{R}$. These states are, of course, observable. The complete sequence of events, along with the free parameters for the probability of occurrence, are given in the tree diagram in the top panel of Figure 1.

The tree diagram shows that as a result of the study trial, the item enters State $L_{S}$ with probability a and State $U_{S}$ with probability $1-a$. The probability of recognition is $p$ if the item is in State $L_{S}$ and $q$ if it is in State $U_{S}$. The probability that the item is in State $L_{R}$ after the recognition test then depends on whether the item was in State $\mathrm{L}_{S}$ or State $\mathrm{U}_{\mathrm{S}}$ after study and whether or not it was recognized. Although we can estimate a from the $\mathrm{P}(\mathrm{URc})$, there are still six parameters $(p, q, v, w, x$, and $y)$, and there are only three independent observable events in the data [e.g., $P(R n)$, $\mathbf{P}(\mathbf{R c})$, and $\mathbf{P}(\mathbf{R n} / \mathbf{R c})]$. This situation can be rectified if we are willing to make some assumptions about how the recognition test might affect recall. Consider first the topmost path, which represents an item in State $L_{S}$ that was also recognized. Since this was an item that was going to be recalled if it had not been present, and since recognizing it should only further increase its strength, this item will be recalled. That is, we can set $v$, the probability of entering State $L_{R}$, equal to 1 . If we are also willing to assume that unrecognized items do not affect the memory trace for the pair, then we can set

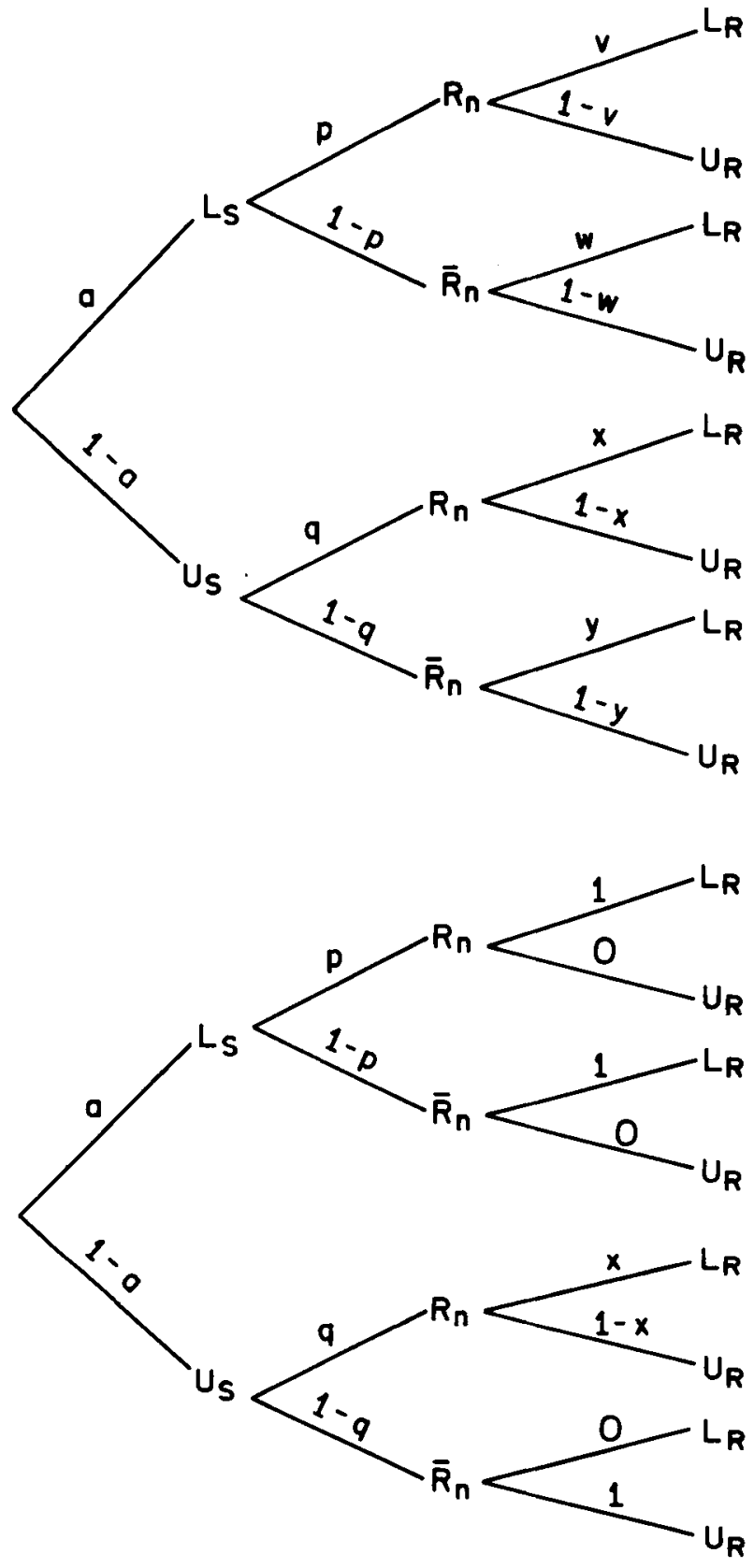

Figure 1. A tree diagram showing the probabilistic effects of study on learning (States $L_{S}$ and $U_{S}$ ), of learning on recognition (States $R_{n}$ and $\bar{R}_{n}$ ), and of recognition on recall (States $L_{R}$ and $U_{R}$ ). In the top panel all parameters are free to vary, and in the bottom panel specific assumptions have been made about some of the parameters. See the text for additional details.

$w=1$ and $y=0$. That is, an item that is assumed to be recallable as a result of the study trial and is not recognized is assumed to still be recallable, and an item that is not recallable after study and is not recognized is assumed to stay not recallable. These assumptions are shown in the tree diagram in the bottom panel of Figure 1. 
Using this tree diagram, we can now write expressions for observable quantities:

$$
\begin{gathered}
P(R c)=a+(1-a) q x \\
P(R n)=a p+(1-a) q \\
P(R n / R c)=[a p+(1-a) q x] /[a+(1-a) q x] .
\end{gathered}
$$

Since $P(U R c)$ is an estimate of $a$, the difference between primed and unprimed recall $(\Delta)$ is an estimate of $(1-a) q x$. Thus, we can rewrite Equation 3 as

$$
\mathrm{P}(\mathrm{Rn} / \mathrm{Rc})=[\mathrm{P}(\mathrm{URc}) \mathrm{p}+\Delta] /[\mathrm{P}(\mathrm{URc})+\Delta] .
$$

\section{GOODNESS OF ENCODING}

Given the constraint on the relationship between $P(R n)$ and $P(R n / R c)$ imposed by the existence of priming effects, what happens if we start with what in effect is the Flexser and Tulving (1978) assumption that the retrieval cues for recognition and recall are independent? We are starting with this assumption in order to evaluate the remainder of their theory, specifically, that variability in the goodness of encoding produces the observed level of dependency between recognition and recall. Our assumption is that unprimed recall and recognition are independent. That is, we will assume that $\mathrm{p}=\mathrm{q}$. We can thus rewrite Equation 4 as

$$
\mathrm{P}(\mathrm{Rn} / \mathrm{Rc})=[\mathrm{P}(\mathrm{URc}) \mathrm{P}(\mathrm{Rn})+\Delta] /[\mathrm{P}(\mathrm{URc})+\Delta] .
$$

Doing this, we can solve for the predicted values of $\mathrm{P}(\mathrm{Rn} / \mathrm{Rc})$ as a function of $\mathrm{P}(\mathrm{Rn}), \mathrm{P}(\mathrm{URc})$, and $\Delta$. The results are given in the next to the last column in Table 1. They should be compared with the observed proportions (see the preceding column) and the predicted results using the Tulving and Wiseman (1975) equation (see the last column). It is clear from these comparisons that if we start with the independence assumption, a substantial proportion of the relationship between $P(R n)$ and $\mathrm{P}(\mathrm{Rn} / \mathrm{Rc})$ is determined by priming. Not all of the relationship is so determined, and it is possible that Flexser and Tulving's (1978) goodness-of-encoding hypothesis might account for the remainder. A principal support for this hypothesis, however, is destroyed by these results. Flexser and Tulving fit the Tulving and Wiseman (1975) function with one free parameter (c) to the data points generated by their model. The parameter estimated by this exercise (.47) was almost identical to the best-fitting parameter using real data (.50). However, our analysis shows that this close correspondence is spurious because much of the relationship between $P(R n)$ and $P(R n / R c)$ is due to priming effects.

\section{TRACE LOSS THEORY}

An examination of the magnitude of the priming effects reported by Begg (1979) permits us to evaluate his hypothesis that trace loss is responsible for the observed dependency between $P(R n)$ and $P(R n / R c)$. As did Flexser and Tulving (1978), Begg in effect starts with the assumption that recognition and recall are independent (the retrieval cues are uncorrelated) and then introduces a measure of dependency by the assumption that neither recognition nor recall can succeed if the memory trace is lost. In evaluating this hypothesis we will also start with the assumption that recognition and recall are independent. We also assume that the memory trace for $A B$ is unaffected by $B$ 's presence on the recognition test if $B$ is not recognized. Begg apparently accepts this assumption, although it is not apparent whether it is as critical for his theoretical thinking as it is for Tulving's.

In his first experiment, Begg (1979) assumed that rote learning would lead to more trace loss than would meaningful learning. Therefore, he predicted greater dependency between recognition and recall after rote learning than after meaningful learning. His measure of dependency was the difference between $P(R n / R c)$ and $\mathrm{P}(\mathrm{Rn})$. This difference was .03 with meaningful learning and .23 with rote learning. Since the priming effect was smaller with rote learning (.08) than with meaningful learning (.10), Begg asserted that priming could not be responsible for the greater dependency observed with rote learning. The amount of dependency introduced by priming, however, is a function not only of the magnitude of the priming effect, but also of the level of recall. Using Equation 5 we actually predict a larger difference between $\mathrm{P}(\mathrm{Rn} / \mathrm{Rc})$ and $\mathrm{P}(\mathrm{Rn})$ for rote learning (.14) than for meaningful learning (.04). It is true that the observed $\mathrm{P}(\mathrm{Rn} / \mathrm{Rc})$ with rote learning (.84) is still substantially above the predicted (.75). This difference, if reliable, may be taken as evidence for the trace loss hypothesis.

The results from the several conditions in Begg's Experiment 3 are shown in Table 2. Begg (1979) noted that there was a significant priming effect [compare $\mathrm{P}(\mathrm{Rc})$ and $\mathrm{P}(\mathrm{URc})]$ and that the proportion recalled conditional on recognition (not shown in Table 2) was almost the same as the proportion recalled $[\mathrm{P}(\mathrm{Rc})]$. From these observations, Begg concluded that since there was priming without dependency between recognition and recall, priming did not necessarily produce a dependency. In Table 2 we have provided the proportion recalled conditional on nonrecognition $[\mathrm{P}(\mathrm{Rc} / \overline{\mathrm{R}} \mathrm{n})]$. This statistic is very similar to the $\mathrm{P}(\mathrm{Rc})$, supporting Begg's conclusion of independence between recognition and recall. Of somewhat more interest, however, is the fact that $P(R c / \bar{R} n)$ is greater than unprimed recall [P(URc)] for six out of the seven conditions and equal to it for the remaining condition. This finding is in sharp contrast to previous results (Begg, 1979, Experiment 1; Bowyer, 1977; Bowyer \& Humphreys, 1979; Humphreys, 1978; Humphreys \& Bowyer, Note 1), which have shown $\mathrm{P}(\mathrm{Rc} / \overline{\mathrm{R}} \mathrm{n})$ to be substantially less than $\mathrm{P}(\mathrm{URc})$. Now, a finding that unprimed recall is worse than primed recall conditional on nonrecognition could 
only occur if one or possibly both of the following statements are true: (1) The memory trace for $A B$ is affected by the presence of $B$ on the recognition test even when $B$ is unrecognized. (2) In this experiment the relationship between recognition and recall without priming is negative. That is, the more likely it is that a word will be recalled, the less likely it is that it will be recognized.

To illustrate the negative relationship in the second alternative, we have estimated $p$ for each condition in Table 2. [Note that $p$ is the $P\left(R n / L_{S}\right)$ and, given our assumptions, is an estimate of what the relationship between recognition and recall would have been without priming.] This estimation is accomplished by solving Equation 4 for $p$ :

$$
\mathrm{p}=\frac{\mathrm{P}(\mathrm{Rn} / \mathrm{Rc})[\mathrm{P}(\mathrm{URc})+\Delta]-\Delta}{\mathrm{P}(\mathrm{URc})} .
$$

The predicted values, with one exception, are less than the unconditional probability of recognition $[P(R n)]$. Thus, the best estimate (assuming that unrecognized words do not affect the memory trace for the pair) of the relationship between recognition and recall indicates a negative relationship for most of the conditions in this experiment. The actual values recorded in Table 2 should not, however, be taken too seriously, as the low levels of recall obtained with most conditions in this experiment yield unstable estimates.

While we can not be certain which of the two alternative assumptions is correct, there is a plausible explanation for a negative relationship between recognition and recall in this experiment. The different conditions in Table 2 represent instructional manipulations. Subjects were either asked to form separate images of the two words in a pair or asked to form a joint image. The separate instructions resulted in slightly better recognition and much poorer recall than did the joint instructions. What happens, then, if, on some occasions, subjects are unable or unwilling to follow these instructions? Suppose subjects given separate instructions occasionally form a joint image and subjects given joint instructions occasionally form separate images. This failure to comply with instructions may occur in any experiment, but it is more likely to occur in this one, as half the subjects received one instruction for their first list and the other for their second list.

In order to determine the effects of adopting an inconsistent coding strategy during the learning of a particular list, we need to make the following assumptions: (1) Within a list, separation imagery produces better recognition and worse recall than does joint imagery, just as it does between lists. (2) For those items learned by a particular strategy, either separation or joint imagery, recall and recognition are independent $(p=q)$. Given these assumptions and the assumption that a subject used both strategies within a list, if a word is recalled, then the probability that it was learned by joint imagery is greater than the unconditional probability that pairs in that list were learned by joint imagery. Conditionalizing on recall thus selects for words that were learned by joint imagery and thus selects for words that are somewhat less likely to be recognized than are unrecalled words.

This observation about the possibility of a negative relationship between recognition and recall does not invalidate the trace loss hypothesis, although it makes it harder to test. Begg (1979) supported his hypothesis by the prediction that the instructional manipulation (separate vs. joint) would not affect the amount of dependency between recognition and recall. If, as we suspect, there was a negative relationship between recognition and recall due to subjects' inconsistent application of the instructions, then it is not possible to predict the expected amount of dependency. The problem is that the amount of dependency observed will depend on the magnitude of the priming effect and the proportion of pairs in the list learned by separate images and by joint images. The fact that the observed proportion recalled was almost the same as the proportion recalled conditional on recognition for both separate and joint instructions must then have been a coincidence.

\section{GENERAL DISCUSSION AND CONCLUSIONS}

Nothing in the analyses presented here implies that for a given pair the recognition and recall cues do not tap independent information, that is, that the fundamental assumption underlying Tulving's approach is not true. However, it is going to be extremely difficult, if not impossible, to determine the actual relationship between recognition and recall. The problem here is that the relationship of interest is at the level of the item or pair. That is, what does knowing that a word was recognized tell us about the probability that it will be recalled? This item-level relationship is obscured by processes operating at a list-wide level. Some of these, such as priming, produce positive covariation between recognition and recall. Others, such as learning different pairs by different methods, can produce negative covariation. Also note that Salzberg (1976) found that stimulus concreteness was positively correlated with response recall but negatively correlated with response recognition. Thus, if stimulus concreteness varies within a list, it will be an additional source of negative covariation between recognition and recall. We can estimate the relationship between recognition and recall in the absence of priming by using Equation 6 . The only substantive assumption that we have to make to obtain this estimate is the commonly accepted assumption that nonrecognized words are not affected by priming, and this assumption is clearly a prerequisite for any attempt to determine the relationship between recognition and recall. Even if we have corrected for priming, however, there still will be a lot of sources capable of producing positive and 
negative covariation (learning pairs by different strategies, the concreteness of the stimulus word, goodness of encoding, trace loss, etc.). The determination of which one is operative in general or in a particular experiment will be almost impossible.

Can we then determine, using the recognition failure paradigm, that recognition and recall are independent, or at least nearly so, as opposed to being dependent, or nearly so? Begg (1979) argued that it would be unlikely that the mechanisms proposed by the dependency theorists (Jones, 1978; Kintsch, 1978; Martin, 1975; Reder et al., 1974) would consistently produce nearly independent results. It is also true that, when corrected for priming effects, most of the results are even closer to being independent. This kind of argument, however, is very hard to evaluate. It may simply be the case that all or almost all of the experiments in this area have used similar procedures and materials. With new procedures and/or materials, the results could strongly favor dependency or a negative relationship. It is also true that our suggestions about negative sources of covariation make the work of the dependency theorist easier. It may not be all that unlikely that positive and negative sources of covariation would roughly balance, producing results that look like independence.

In conclusion, we are extremely pessimistic about the possibility that one can start with the assumption that the retrieval cues for recognition and recall are independent and go on to identify a particular source of positive covariation (goodness of encoding, trace loss, etc.). We are also pessimistic about the possibility of determining at the level of an individual pair whether recognition and recall are basically independent or dependent. These pessimistic conclusions, of course, only apply to the recognition failure paradigm and the use of conditional probabilities to elucidate the recognition-recall relationship.

\section{REFERENCE NOTE}

1. Humphreys, M. S., \& Bowyer, P. A. Familiarization trials on one member of a study pair: The effects on the other member. Manuscript submitted for publication, 1979.

\section{REFERENCES}

BEGG, I. Trace loss and the recognition failure of unrecalled words. Memory \& Cognition, 1979, 7, 113-123.

BoWyer, P. Priming of unrecognized items on a subsequent cued recall test. Unpublished master's thesis, Northwestern University, 1977.

Bowyer, P. A., \& Humphreys, M. S. The effect of a recognition test on a subsequent cued recall test. Journal of Experimental Psychology: Human Learning and Memory, 1979, 5, 348-359.

Flexser, A. V., \& Tulving, E. Retrieval independence in recognition and recall. Psychological Review, 1978, 85, 153-171.

HUMPHREYS, M. S. Item and relational information: A case for context independent retrieval. Journal of Verbal Learning and Verbal Behavior, 1978, 17, 175-187.

Jones, G. V. Recognition failure and dual mechanisms in recall. Psychological Review, 1978, 85, 464-469.

KINTSCH, W. More on recognition failure of recallable words: Implications for generation-recognition models. Psychological Review, 1978, 85, 470-473.

Martin, E. Generation-recognition theory and the encoding specificity principle. Psychological Review, 1975, 82, 150-153.

Postman, L. Tests of the generality of the principle of encoding specificity. Memory \& Cognition, 1975, 3, 663-672.

Reder, L. M., Anderson, J. R., \& Bjork, R. A. A semantic interpretation of encoding specificity. Journal of Experimental Psychology, 1974, 102, 648-656.

Salzberg, P. M. On the generality of encoding specificity. Journal of Experimental Psychology: Human Learning and Memory, 1976, 2, 586-596.

Tulving, E. Ecphoric processes in recall and recognition. In J. Brown (Ed.), Recall and recognition. London: Wiley, 1976.

Tulving, E., \& Thomson, D. M. Encoding specificity and retrieval processes in episodic memory. Psychological Review, 1973, 80, 352-373.

Tulving, E., \& Wiseman, S. Relation between recognition and recognition failure of recallable words. Bulletin of the Psychonomic Society, 1975, 6, 79-82.

Vining, S. K., \& Nelson, T. O. Some constraints on the generality and interpretation of the recognition failure of recallable words. American Journal of Psychology, 1979, 92, 257-276.

Watkins, M. J., \& Tulving, E. Episodic memory: When recognition fails. Journal of Experimental Psychology: General, 1975, 104, 5-29.

Wiseman, S., \& Tulving, E. Encoding specificity: Relation between recall superiority and recognition failure. Journal of Experimental Psychology: Human Learning and Memory, 1976, 2, 349-361.

(Received for publication July 6, 1979; revision accepted October $31,1979$. ) 